\title{
THE EXPRESSION OF LONG LASTING AFTERDISCHARGE BY ISOLATED APLYSIA BAG CELL NEURONS ${ }^{1}$
}

\author{
L. K. KACZMAREK AND F. STRUMWASSER ${ }^{2}$
}

Division of Biology, California Institute of Technology, Pasadena, California 91125

\begin{abstract}
The cluster of neuropeptidergic bag cells in the abdominal ganglion of Aplysia is able to generate a long lasting afterdischarge of compound action potentials following brief electrical stimulation of the pleurovisceral connective nerve or extracellular addition of cyclic adenosine $3^{\prime}: 5^{\prime}$-monophosphate (cAMP) analogues. We have now examined the response of individual cultured bag cells to the cAMP analogue, 8-benzylthio-cAMP, applied extracellularly or intracellularly.

In common with bag cells in an intact cluster, individual cultured bag cells respond to extracellular application of $0.5 \mathrm{~mm} 8$-benzylthio-cAMP by generating a long lasting discharge of action potentials (mean duration $=123 \mathrm{~min}$ ). The discharge, in cultured cells, started after a mean delay of 26 min following addition of the cAMP analogue. This long delay suggests an intracellular site of action for the cAMP analogue. The discharge could be blocked by cobalt ions but not by tetrodotoxin. The frequency of the 8-benzylthio-cAMP-induced discharge often could be increased by brief repetitive intracellular stimulation. Moreover, if, after addition of benzylthio-cAMP, discharge was prevented by a hyperpolarizing bias current, a short burst of spikes ( 5 to $15 \mathrm{sec}$ ), produced by releasing the hyperpolarization, could induce a prolonged afterdischarge in the presence of the bias current.

The onset of the discharge induced in cultured cells by 8-benzylthio-cAMP was preceded by an increase in membrane resistance (mean increase $=68 \%$ ) and a decrease in the threshold for spikes evoked by depolarizing current pulses. The width of single action potentials evoked by a threshold depolarizing pulse of constant current was augmented following 8-benzylthio-cAMP. Moreover, frequency-dependent spike broadening also was enhanced markedly. Fully broadened action potentials generated by repetitive current pulses $(\sim 1$ pulse/sec) were approximately double the width of controls and were characterized by a prominent shoulder on their falling phase. This enhancement of spike broadening preceded the onset of the discharge. In 3 out of 17 experiments, a regenerative hyperpolarizing response was also observed following 8-benzylthio-cAMP. This transient response was associated with an increase in membrane conductance. Direct intracellular microinjection of 8benzylthio-cAMP into bag cell neurons also enhanced spike genesis, increased membrane resistance, and produced subthreshold oscillations in membrane potential and spontaneous discharge.

The response of cultured bag cells to 8-benzylthio-cAMP qualitatively resembles the response of intact bag cell systems to either cAMP analogues or electrical stimulation. Quantitative differences exist, however, particularly in the frequency of firing and mode of onset of discharge in these two systems. The response of the cultured bag cells is consistent with the hypothesis that cAMP induces a decrease in the membrane conductance to potassium ions.
\end{abstract}

It is known that the principal function of the peptidergic bag cell neurons of the abdominal ganglion of Aplysia is to "command" the onset of egg laying and its associated behavior by releasing their neurosecretory product, egg-laying hormone (ELH), during a long lasting afterdischarge (Kupfermann, 1970; Kupfermann and Kandel, 1970; Strumwasser et al., 1980; Stuart et al.,

' This work was supported by National Institutes of Health Grant NS 13896 to F. S. We thank Mr. John Scotese and Mr. Daniel P. Viele for preparing the primary cultures of bag cells used in these studies.

${ }^{2}$ To whom reprint requests should be addressed.
1980). In the absence of external stimulation, the bag cells within an intact abdominal ganglion have relatively high resting potentials and show no spontaneous activity. Following either brief electrical stimulation of a presumed afferent pathway in the pleuroabdominal connectives or the extracellular application of peptides from the atrial gland (Dudek and Blankenship, 1977a; Heller et al., 1979, 1980; Kaczmarek et al., 1978; Kupfermann and Kandel, 1970), the bag cells depolarize and discharge repetitively, and in synchrony, for a duration of about 30 min, after which, the cells become relatively refractory to further stimulation (Dudek and Blankenship, 1977b; 
Kaczmarek et al., 1978; Kupfermann and Kandel, 1970).

A variety of lines of evidence suggests that these electrical properties are controlled by the intracellular concentration of adenosine $3^{\prime}: 5^{\prime}$-monophosphate (cAMP) (Kaczmarek et al., 1978). Initiation of afterdischarge is associated with an $\sim 200 \%$ increase in cAMP levels within the bag cells which peaks around 2 min into afterdischarge and, thereafter, declines to control levels. The afterdischarge may be initiated by a number of cAMP analogues (Kaczmarek et al., 1978) including 8-benzylthio-cAMP, 8-methylthio-cAMP, and $o-\mathrm{NO}_{2}$-benzylcAMP ester (whereas analogues of guanosine $3^{\prime}: 5^{\prime}$-monophosphate are without effect; L. K. Kaczmarek and J. Nerbonne, unpublished results). In addition, the duration of the afterdischarge may be greatly prolonged by phosphodiesterase inhibitors (Kaczmarek et al., 1978).

Our present studies are an attempt to determine how much of the afterdischarge property is intrinsic to single bag cells isolated in cell culture (Kaczmarek et al., 1979; Strumwasser et al., 1978). In the intact abdominal ganglion, the processes of the bag cell neurons form a complex network that is coupled by gap junctions (Kaczmarek et al., 1979). Cell culture offers the advantage of making electrical measurements and intracellular injection possible without the complications of the electrical, and metabolic, coupling that results from such interconnections (Kaczmarek et al., 1979; Blankenship and Haskins, 1979). We have studied the effects of the membranepermeant, phosphodiesterase-resistant cAMP analogue, 8-benzylthio-cAMP (8BT-cAMP) (Meyer and Miller, 1974; Treistman and Levitan, 1976) on the properties of isolated bag cell neurons and show that such isolated neurons respond to this analogue with enhanced spike electrogenesis, increased membrane resistance, and the onset of oscillations in membrane potential that drive a long lasting discharge. Moreover, in the presence of this analogue, a brief bout of spike production may generate a prolonged afterdischarge. We suggest that these changes are the result of a long lasting decrease in potassium ion conductance.

\section{Materials and Methods}

Aplysia californica was collected locally (southern California coast) by Mr. John Scotese. The animals were kept at $14^{\circ} \mathrm{C}$ and all electrophysiological experiments were carried out at this temperature. Abdominal ganglia were dissected out and incubated at $22^{\circ} \mathrm{C}$ for $6 \mathrm{hr}$ in filtered seawater containing $1.25 \%$ neutral protease with neomycin sulfate $(100 \mu \mathrm{g} / \mathrm{ml})$ (Kaczmarek et al., 1979; Strumwasser et al., 1978). After removing the connective tissue capsules, the bag cells were disaggregated using a Pasteur pipette and seeded into $35-\mathrm{mm}$ Falcon tissue culture dishes containing modified L-15 medium (Leibovitz, 1963) obtained from Gibco and made up in artificial seawater containing $15 \mathrm{~mm}$ Hepes (4-(2-hydroxyethyl)-1piperazine-ethanesulfonic acid, $\mathrm{pH} 7.8$ ) and neomycin sulfate $(50 \mu \mathrm{g} / \mathrm{ml})$. The cells rapidly attached to the bottom of the culture dishes and within 2 or 3 days, had developed elaborate neuritic branches (Strumwasser et al., 1978, 1980). Electrophysiological experiments usually were carried out within 1 week of seeding.

Glass microelectrodes were pulled on a Brown-Flaming electrode puller and filled with $2 \mathrm{~m}$ potassium citrate. These electrodes had resistances of 30 to 100 megohms. Cultured bag cell neurons were penetrated, under visual control, on a Leitz Diavert microscope. Recording of membrane potential was through an M701-WPI electrometer and the intensity of current applied to depolarize or hyperpolarize the cell was monitored using either the current monitor circuit of the electrometer or an independent virtual ground circuit. When cells were impaled with a single electrode, which was used for both voltage recording and passage of current, the input resistance of the cells was estimated by measuring the maximum voltage displacements to a range of small hyperpolarizing currents after correction for bridge imbalance. In some experiments, however, the cells were penetrated with a second microelectrode which was used for the passage of imposed currents.

For pressure microinjection of $8 \mathrm{BT}$-cAMP, the tips of microelectrodes were brushed against a ground glass plate, to produce final tip diameters of $\sim 1.0 \mu \mathrm{m}$. These electrodes were filled with $20 \mathrm{~mm} 8 \mathrm{BT}$-cAMP, $0.3 \mathrm{M} \mathrm{KCl}$ and mounted in an electrode holder connected to a cylinder of $\mathrm{N}_{2}$ gas so that pulses of pressure (15 to $45 \mathrm{psi}$, $2 \mathrm{sec}$ to $2 \mathrm{~min}$ ) could be applied to the interior of the electrodes under control of a solenoid-operated threeway valve (McCaman et al., 1977). Pressure injection into bag cells was carried out as described elsewhere (Kaczmarek et al., 1980).

To achieve the final concentrations of pharmacological agents used in this study, 30 to $300 \mu \mathrm{l}$ of a more concentrated solution were added directly to the $3 \mathrm{ml}$ of $\mathrm{L}-15$ medium bathing the cultured cells in a $35-\mathrm{mm}$ Falcon culture dish. 8BT-cAMP was obtained from ICN (standard and high pressure liquid chromatography (HPLC) pure grades).

\section{Results}

The electrophysiological effects of the addition of 0.5 mM 8BT-cAMP to the extracellular medium of cultured bag cells include the onset of spontaneous spike discharge and the ability to afterdischarge following brief intracellular stimulation. The discharges were preceded by an increase in membrane resistance with a decrease in the spike threshold and enhanced spike broadening in response to repetitive current pulses. In a minority of cases, a regenerative hyperpolarizing response was also observed. These changes will be dealt with individually.

Onset of repetitive discharge. Although action potentials may be evoked in cultured bag cells by threshold depolarizing current pulses (0.06 to $0.28 \mathrm{nA})$, such cells normally do not exhibit spontaneous spike activity except for transient spiking that sometimes can be seen immediately following microelectrode penetration. Extracellular application of 8BT-cAMP $(0.5 \mathrm{~mm})$, however, systematically induced a prolonged discharge in these cells. Figure $1 a$ shows chart records of spiking activity elicited by this cAMP analogue. Of 17 cells that were judged as healthy cultured cells by the criteria of resting potential $(>35 \mathrm{mV}$, mean resting potential $=43.2 \pm 2.4 \mathrm{mV}$ (SEM)) and the ability to produce overshooting action potentials, 14 responded to $8 \mathrm{BT}$-cAMP by discharging in this way. For all of these cells, the onset of the discharge was 
gradual as is shown by the plot of firing rate, as a function of time following 8BT-cAMP application, for a representative cell (Fig. 1b).The maximal firing rate observed in these experiments was 10 to 30 spikes $/ \mathrm{min}$. The mean duration of the discharges was $123 \pm 33 \mathrm{~min}$ (SEM, $N$ $=7$ ) and the mean latency to onset following the addition of 8BT-cAMP was $26.2 \pm 5.6 \mathrm{~min}(\mathrm{SEM}, N=14)$. We could observe no consistent change in the resting potential of the cells prior to the onset of the discharge, although small changes would have been difficult to detect against base line drift over these time periods.

The 8BT-cAMP-induced discharge occurred both in fully developed cells that had been in culture for several days and in more recently seeded cells that had not yet extended elaborate neuritic branching patterns. The onset of discharge was always associated with the onset of subthreshold oscillations of the membrane potential. These oscillations varied, irregularly, in amplitude and appeared to be related to smaller graded amplitude spikes. The oscillations can be seen clearly in Figure $1 a$ and they generally persisted for some hours following the end of the discharge. Since they were observed both in cells with elaborate branching patterns and in freshly seeded cells, they are unlikely to represent distal neuritic spikes although this explanation cannot be discounted entirely since even apparently aneuritic cells may be

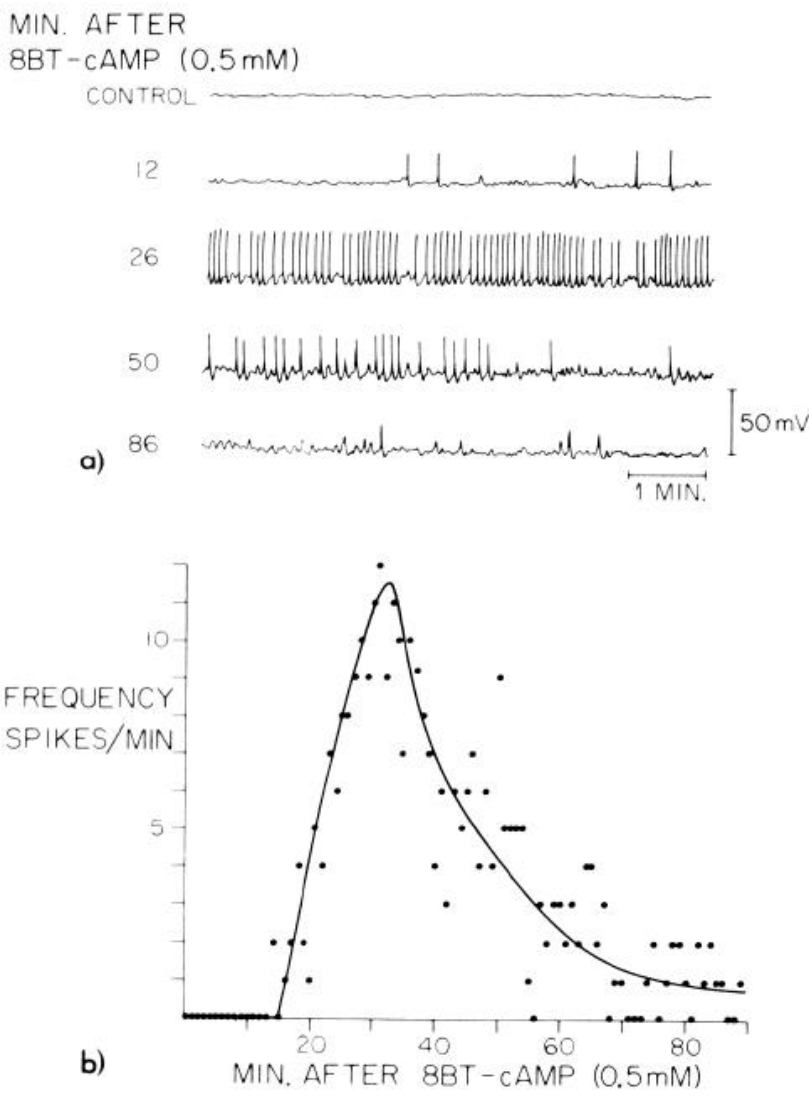

Figure 1. The onset of repetitive discharge in cultured bag cells in response to $8 \mathrm{BT}$-cAMP $(0.5 \mathrm{mM})$. The upper half of the figure shows intracellular records taken at various stages following the addition of 8BT-cAMP. The lower half plots the number of spikes in successive 1-min epochs for the same experiment.
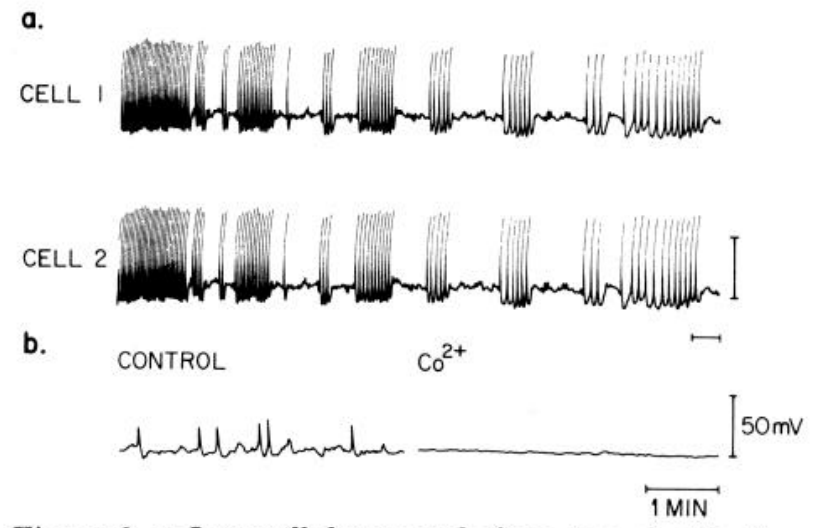

Figure 2. a, Intracellular records from two electrically coupled bag cells in cell culture after repetitive discharge had been induced by $8 \mathrm{BT}$-cAMP $(0.5 \mathrm{~mm})$. It can be seen that action potentials and the smaller fluctuations in membrane potential are synchronous in the two cells. $b$, The control shows the fluctuations in membrane potential and partial spikes observed during 8BT-cAMP-induced discharge. This tracing was taken immediately before the addition of $12 \mathrm{mM} \mathrm{CoCl} 2$ to the extracellular medium, which, as shown on the right, immediately abolished the fluctuations.

attached to the substrate by fine processes. Both the discharge and the oscillations were unaffected by tetrodotoxin $\left(5 \times 10^{-5} \mathrm{M}\right)$ but could be blocked by $12 \mathrm{~mm}$ $\mathrm{CoCl}_{2}$ (Fig. 2b), suggesting that they are $\mathrm{Ca}^{2+}$ dependent.

We have described electrical coupling between bag cells in cell culture previously (Kaczmarek et al., 1979). In one experiment, we penetrated two coupled bag cells (coupling ratio, 0.79) and recorded from both cells during an 8BT-cAMP-induced discharge. Both cells discharged synchronously and Figure $2 a$ shows that the smaller membrane potential oscillations are also synchronous in the two cells, which implies that these low frequencies are propagated effectively, probably by a combination of active propagation and passive spread.

Action potential-evoked afterdischarge. During discharges induced by $8 \mathrm{BT}$-cAMP, it was noted that a brief train of threshold depolarizing pulses (10 to 15 pulses, 1 / $\mathrm{sec}$ ) could sometimes increase the firing rate of a cell for several minutes following stimulation (Fig. $3 a$ ). This suggested that a brief train of action potentials in isolated bag cell neurons could produce an afterdischarge. We use the term "afterdischarge" to describe a prolonged discharge that is triggered by brief repetitive stimulation. To investigate this effect in more detail, three additional cells were exposed to 8BT-cAMP $(0.5 \mathrm{~mm})$ after which a hyperpolarizing bias current was applied. The magnitude of this current ( 0.06 to $0.08 \mathrm{nA}$ ) was adjusted so as to just prevent the occurrence of the $8 \mathrm{BT}$-cAMP-induced discharge. The bias current then was released for a period of 10 to $15 \mathrm{sec}$, during which, the cells fired repetitively. In all three cells, a prolonged (12- to 20-min) afterdischarge followed in the presence of the current that had suppressed repetitive discharge previously. Figure $3 b$ shows such an afterdischarge for one of the cells. The onset of afterdischarge always occurred after a delay of 10 to $40 \mathrm{sec}$ following the burst of spikes produced by releasing the bias current (see Fig. $3 b$ ). In each of the three cells, this phenomenon could be repeated several 
times, although subsequent afterdischarges were shorter in duration (1.5 to $8.0 \mathrm{~min}$ ). Finally, permanent release of the bias current reinstated the long lasting $8 \mathrm{BT}$-cAMPinduced discharge described above.

Neither repetitive depolarizing stimulation nor the imposition and release of a hyperpolarizing bias current could induce afterdischarge prior to the addition of $8 \mathrm{BT}$ cAMP.

Membrane resistance. $8 \mathrm{BT}$-cAMP induced a progressive increase in the apparent resistance of bag cell membranes as judged by the size of voltage displacements caused by hyperpolarizing and depolarizing current pulses of constant intensity. Figure $4, A$ and $B$, shows these effects for two different cells. At the time of onset of the 8BT-cAMP-induced discharge, the mean membrane resistance in nine cells was found to have increased by $68 \pm 23 \%$ (SEM). This resistance increase resulted in a decreased current threshold for spike genesis so that depolarizing current pulses that were subthreshold prior to $8 \mathrm{BT}$-cAMP could frequently generate full action potentials (Fig. 4, $A$ and $B$ ). For depolarizing current pulses that were suprathreshold prior to $8 \mathrm{BT}$-cAMP, the latency from the onset of the pulse to spike genesis was reduced markedly after the cAMP analogue (Fig. 5). The time course of this reduction in latency, following $8 \mathrm{BT}$ -
cAMP, is shown in Figure $6 b$ and indicates that this effect occurs prior to the onset of the induced discharge. The increase in input resistance and reduction in latency of evoked spikes also preceded the ability to afterdischarge in response to repetitive stimulation.

As mentioned above, 3 out of 17 cells failed to discharge in response to $8 \mathrm{BT}$-cAMP. In two of these cases, we determined that an increase in membrane resistance did take place following 8BT-cAMP. Moreover, prior to addition of the analogue, the response of both cells to any prolonged suprathreshold depolarizing pulse ( 2 to $5 \mathrm{sec}$ ) was a phasic response of only 2 or 3 action potentials at the onset of the pulse. In both cases, following $8 \mathrm{BT}$ cAMP, the cells responded to these prolonged depolarizing pulses by firing repetitively with 9 to 25 action potentials and with very little adaptation of firing rate throughout the pulse. Thus, although these cells failed to discharge spontaneously, 8BT-cAMP did induce changes in their membrane properties in the direction of increased excitability.

Frequency-dependent spike broadening. Depolarizing current pulses, when applied repetitively at certain frequencies, may cause the action potentials of cultured bag cells to increase in amplitude and in duration (Strumwasser et al., 1981). The effect of 8BT-cAMP (0.5 mM)

a)

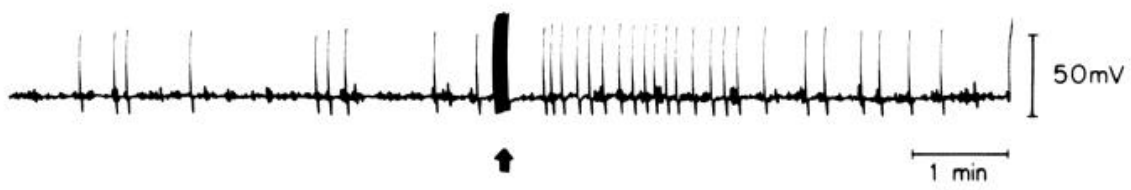

b)
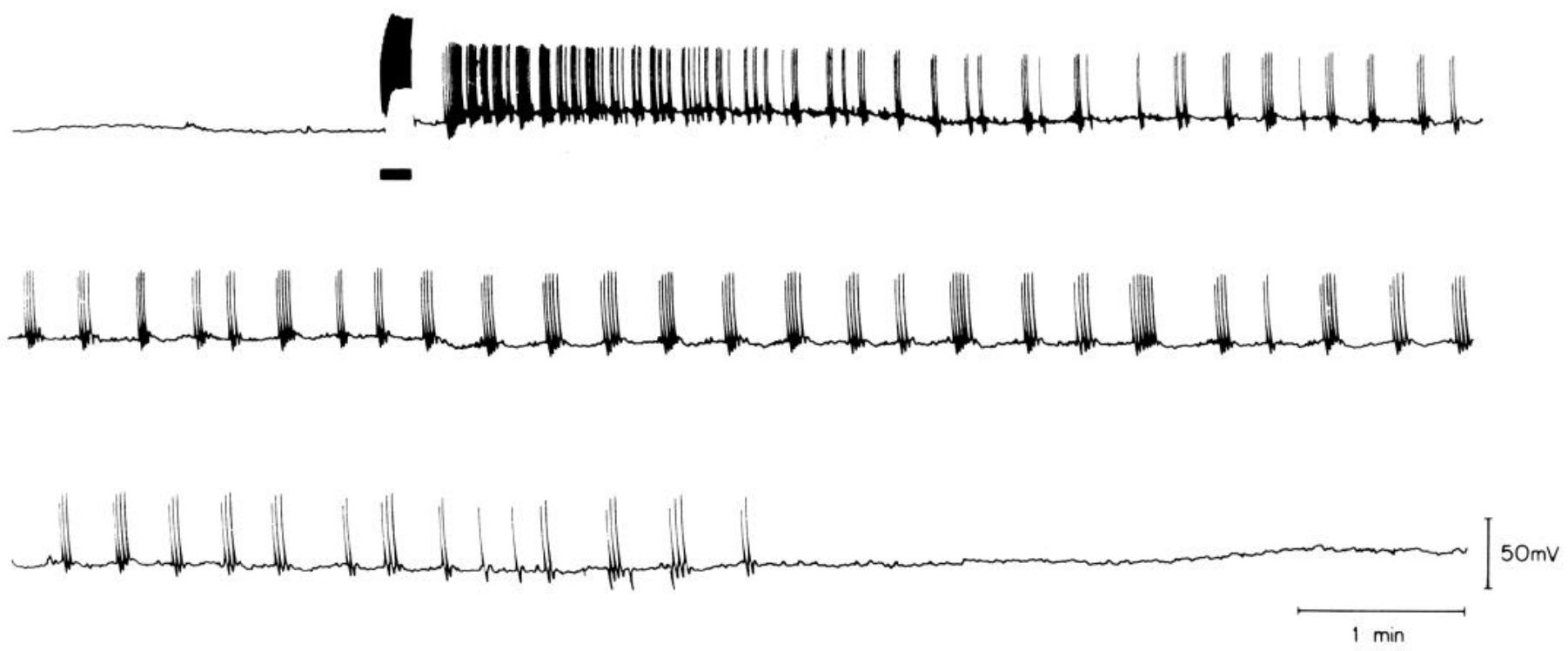

Figure 3. $a$, An intracellular record from a bag cell neuron in cell culture after repetitive discharge had been induced by $8 \mathrm{BT}$ cAMP. At the time marked by the arrow, a series of 10 depolarizing current pulses (250 msec, $0.25 \mathrm{nA}, 0.83 \mathrm{pulses} / \mathrm{sec})$ was applied to the cell. Following the pulses, an increase in the frequency of discharge was observed. $b$, Afterdischarge in a bag cell neuron in cell culture. After addition of $8 \mathrm{BT}$-cAMP $(0.5 \mathrm{~mm})$ to the extracellular medium, a hyperpolarizing current $(0.06 \mathrm{nA})$ was applied to the cell to prevent the onset of a repetitive discharge. For the duration indicated by the bar, this bias current was released, resulting in repetitive firing by the cell. After reapplication of the bias current, a prolonged afterdischarge was generated. 
CONTROL

A
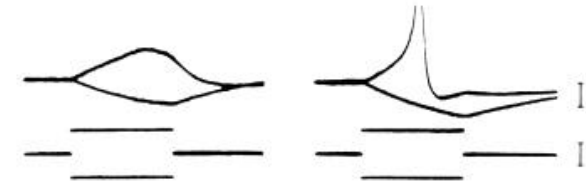

B
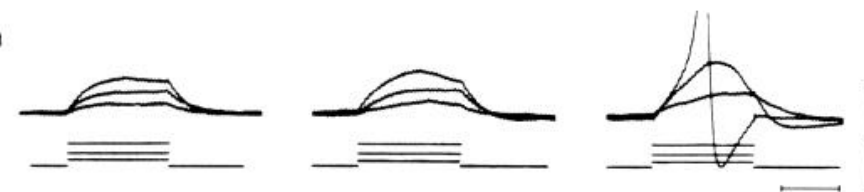

CALIBRATION IOMV, O.InA, $250 \mathrm{mSEC}$

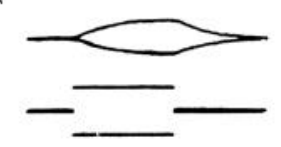

Figure 4. The increase in membrane resistance induced in cultured bag cells by 8 BT-cAMP $(0.5 \mathrm{~mm})$. A, Superimposed traces of the membrane potential responses (upper traces) to hyperpolarizing or depolarizing current pulses of constant intensity (lower traces). The left set of tracings shows the response prior to the addition of 8BT-cAMP. The center set shows the response $8 \mathrm{~min}$ after addition of the cAMP analogue and the right set of tracings shows the response after $40 \mathrm{~min}$. $B$, This shows superimposed tracings of membrane potential responses (upper traces) to three different intensities of depolarizing current pulses (lower traces). The left set shows the control responses while the center and right show the responses 14 and $80 \mathrm{~min}$, respectively, after addition of 8BT-cAMP. In the right set of traces for both $A$ and $B$, action potentials have been truncated by the oscilloscope screen.

on these cells is to enhance spike broadening markedly as is shown in Figure 5. The control column shows superimposed traces of action potentials in response to 10 constant current pulses given at different frequencies. At the lowest frequency ( 1 pulse every $10 \mathrm{sec}$ ), no spike broadening occurs and the traces superimpose one on another. At higher frequencies, the traces corresponding to the later pulses in the train of 10 pulses show that the action potentials become progressively larger and broader. This effect is most evident at the highest frequencies (periods of $1 \mathrm{sec}$ and $600 \mathrm{msec}$ ). Following $8 \mathrm{BT}$-cAMP $(0.5 \mathrm{~mm})$, the frequency dependence of spike broadening is unchanged as is shown in the second column of Figure 5. The shape of the spikes is altered, however. Even at low frequencies at which frequencydependent broadening does not occur, the spikes are significantly broader following $8 \mathrm{BT}$-cAMP. At the higher frequencies, spike broadening is enhanced. The broadened action potentials have a prominent shoulder on their falling phase and their width is approximately double that of the fully broadened spikes in the controls. The time course for the onset of enhanced spike broadening is shown in Figure $6 a$ by plotting the width of successive action potentials in a train of $1 / \mathrm{sec}$ pulses as a function of the time following $8 \mathrm{BT}$-cAMP addition to the culture dish. The width of the action potentials was measured at $40 \mathrm{mV}$ positive to the resting potential of this cell (49 $\mathrm{mV})$. The figure shows that spike broadening is profoundly enhanced prior to the onset of the discharge but that some further enhancement also occurs during the discharge.

Enhancement of spike broadening also occurred prior to the ability to afterdischarge in those experiments in which afterdischarges were evoked by repetitive stimulation following 8BT-cAMP addition.

Regenerative hyperpolarizing response. Before the addition of 8BT-cAMP, the normal response of cultured bag cells to hyperpolarizing current pulses is a passive linear membrane response. In some of these experiments, a nonlinear component to the hyperpolarizing response could be detected following 8BT-cAMP $(0.5 \mathrm{~mm})$. This component resulted in a markedly slowed return of the voltage trajectory to the resting potential following the end of the current pulse. This nonlinear component was particularly evident in three experiments which provided a regenerative response to brief hyperpolarization. The form of this response is shown in Figure $7 a$. A brief hyperpolarizing current pulse produced a hyperpolarizing voltage trajectory. If the current pulse was sufficiently large, the voltage trajectory continued to hyperpolarize, after the end of the pulse to reach a fixed "plateau" at which the potential remained for many seconds with only a very slow depolarizing drift. The end of the response occurred with an abrupt return to the resting potential. In these three experiments, the regenerative hyperpolarizing response also occurred spontaneously during or after the 8BT-cAMP-induced discharge and sometimes could be triggered by a spike afterpotential (Fig. $7 b$, lower trace). Using constant current pulses of low amplitude that did not trigger this response, it could be shown that the response was associated with a decrease in membrane resistance (Fig. $7 a$, right panel).

Intracellular injection of $8 B T \cdot c A M P$. Pressure injection was used to introduce 8BT-cAMP intracellularly into four cultured bag cell neurons. The penetration of cultured bag cell neurons with the large tip diameter micropipettes required for pressure microinjection resulted in the cells having lower resting potentials (mean $=30.0 \mathrm{mV}$ ) than those used for the extracellular application of 8BT-cAMP $($ mean $=43.2 \mathrm{mV})$. Nevertheless, three of these cells responded to the injections with an increase in membrane resistance and, of these, two showed enhanced spike electrogenesis in response to constant depolarizing current pulses (Fig. $8 A$ ). The enhancement of spikes differed slightly from that observed with extracellular addition of 8BT-cAMP in that, in addition to the increase in broadening with repetition, the amplitude and slope of the rising phase of the action potentials was increased relative to the pre-injection controls (Fig. 8A).

Only one of the cells injected with 8BT-cAMP generated subthreshold oscillations and discharge following injection (Fig. 8B). An additional two cells, however, started to discharge repetitively immediately following penetration with the large tipped 8BT-cAMP-containing microelectrodes. Subthreshold oscillations and occasional discharge continued for the duration of the experiments ( $>1 \mathrm{hr}$ ), presumably due to leakage of 8BT-cAMP from the micropipettes. The subthreshold oscillations remained constant in amplitude $(4.5 \mathrm{mV})$ and frequency $(\sim 1 / \mathrm{sec})$. Both their amplitude and frequency could be increased by applying depolarizing transmembrane currents and decreased by hyperpolarizing currents (Fig. $8 C)$. 

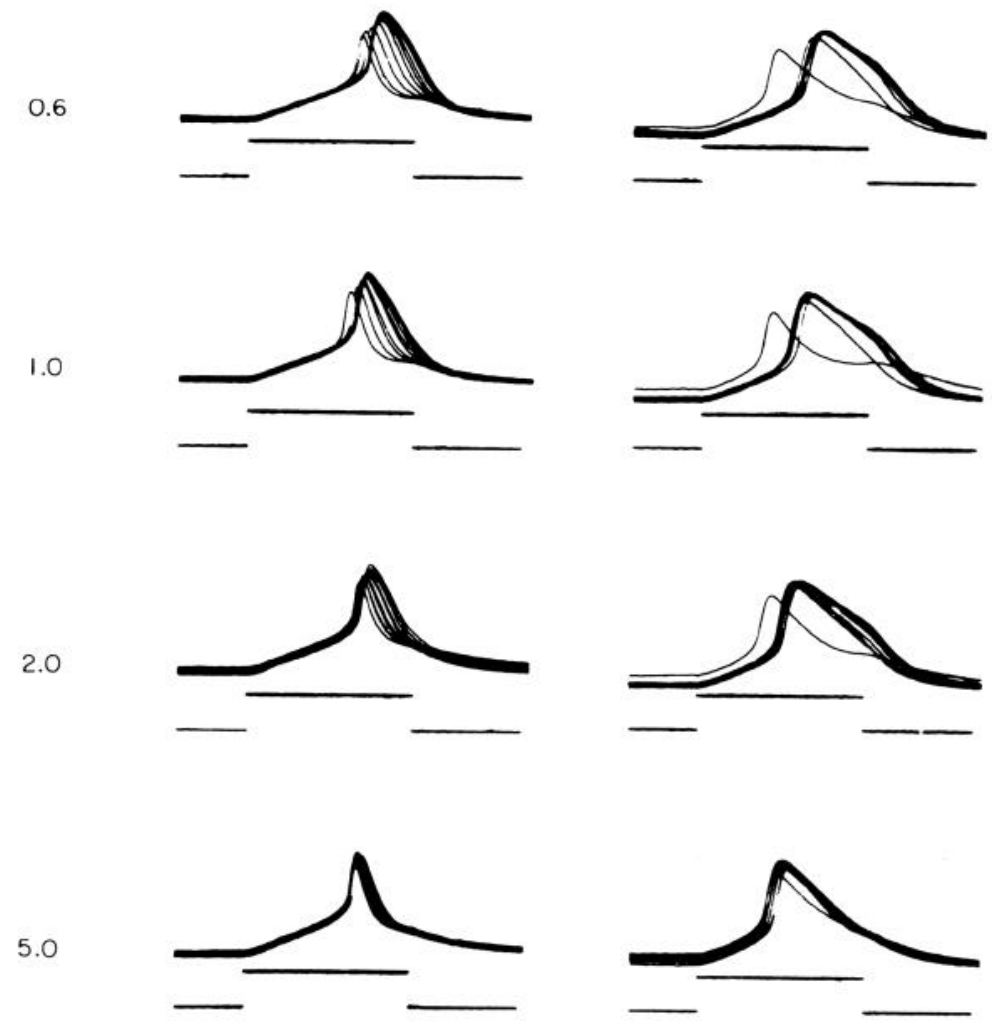

10.0
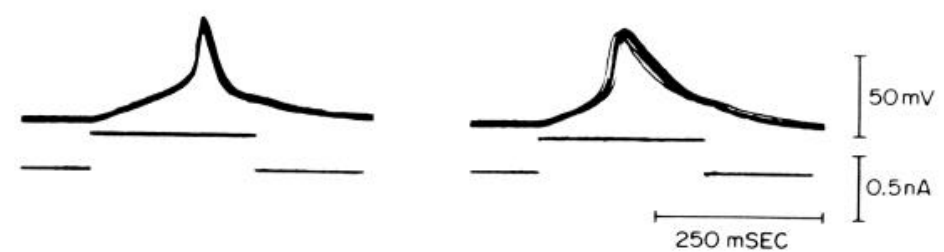

Figure 5. The effect of 8BT-cAMP on frequency-dependent spike broadening. Each set of traces in the left column shows 10 superimposed action potentials (upper traces) evoked by consecutive depolarizing current pulses (lower traces). The period between current pulses is indicated to the left of each set of traces. The right column shows the response of the same cell to repetitive current pulses of the same intensity and period following exposure to 8BT-cAMP (0.5 mM, 100 $\min )$.

\section{Discussion}

We have shown previously that the intact bag cell cluster responds to brief electrical stimulation of a presumed synaptic pathway by a prolonged 30 -min afterdischarge which is correlated with a 3 -fold increase in cAMP by 2 min into afterdischarge (Kaczmarek et al., 1978). The cAMP analogue, 8-benzylthio-cAMP, initiates a discharge in the intact bag cell cluster that is similar to the electrically induced afterdischarge in that there is initially a high frequency discharge ( 3 to 6 spikes/sec) lasting for 1 to $1 \frac{1}{2} \mathrm{~min}$ followed by a more prolonged lower frequency $(\sim 20$ to 2 spikes/min $)$ discharge (Kaczmarek et al., 1978; Kupfermann and Kandel, 1970; Strumwasser et al., 1981). We postulated, based on these results and the finding that certain phosphodiesterase inhibitors could prolong afterdischarge significantly, that the increase in intracellular cAMP induces a long lasting increase in membrane excitability which generates the afterdischarge.

We now have shown that afterdischarge, the ability to respond to brief stimulation with a subsequent prolonged discharge, is an intrinsic property of isolated bag cell neurons which is expressed after exposure to 8BT-cAMP. The ability to discharge spontaneously in response to 8BT-cAMP is also intrinsic to these isolated cells. Qualitatively, the effects of $8 \mathrm{BT}$-cAMP on cultured bag cell neurons resemble those seen in the intact bag cell cluster. The latency between 8BT-cAMP addition and the onset of discharge in cultured cells (between 8 and $55 \mathrm{~min}$ ) is 

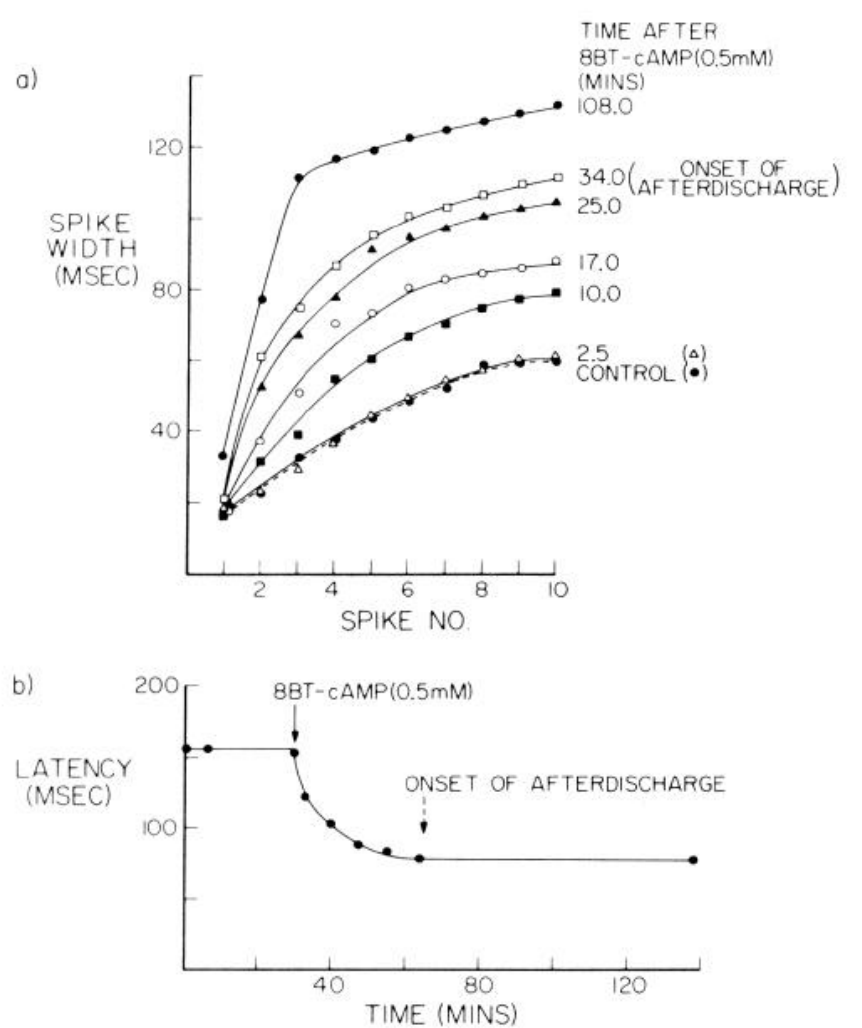

Figure 6. $a$, The time course of the enhancement of frequency-dependent spike broadening following extracellular application of $8 \mathrm{BT}$-cAMP $(0.5 \mathrm{~mm})$. Ten depolarizing current pulses ( $250 \mathrm{msec}, 0.25 \mathrm{nA}$ ) were applied to a cultured bag cell at a frequency of $1 / \mathrm{sec}$. The width of successive action potentials was measured at a potential $40 \mathrm{mV}$ positive relative to the resting potential of the cell $(49 \mathrm{mV})$ and plotted as a function of pulse number. The graphs for control and for the responses at various times after addition of $8 \mathrm{BT}$-cAMP are indicated on the right of the figure. The term, onset of afterdischarge, indicates the onset of repetitive discharge at $34 \mathrm{~min}$ of exposure to $8 \mathrm{BT}$-cAMP. $b$, This plots the latency from the onset of a current pulse (250 msec, $0.25 \mathrm{nA})$ to the point of inflection at the base of the first action potential as a function of time following 8BT-cAMP addition for the same cell as in $a$.

comparable to that for the onset of afterdischarge in intact bag cell clusters (between $3^{1 / 2}$ and $28 \mathrm{~min}$ ) (Kaczmarek et al., 1978). Moreover, the onset of discharge in both systems is associated with a profound increase in spike broadening (Strumwasser et al., 1981). There are, however, some quantitative differences between the response of individual cultured bag cells to extracellular application of 8BT-cAMP and that of bag cells in an intact cluster. In the cultured cells, not subject to any bias current, the onset of the discharge is more gradual than in the intact system and the frequency of firing ( 1 to 15 spikes/min) never attained the high frequencies observed during the 1st min of an afterdischarge in the intact system, although they were comparable to the rates of firing observed later in an intact cluster. Moreover, the mean duration of the discharges in culture (123 min) was longer than that of an intact preparation $(\sim 30 \mathrm{~min})$. These differences could arise either from the absence of cell-cell interactions in culture or from meta-

bolic differences between the cultured and intact systems.

We have shown that the onset of 8BT-cAMP-induced discharge in isolated bag cell neurons is associated with an increase in membrane resistance and an enhancement of frequency-dependent spike broadening. Although alternative explanations are possible, we feel that a likely interpretation of these effects is that 8BT-cAMP induces a net decrease in potassium ion conductance across the cell membrane. A decrease in potassium conductance would be consistent with the increase in membrane resistance and the increased excitability of the cells. A progressive inactivation of an outward potassium current has been shown to produce frequency-dependent broadening of action potentials in other molluscan neurons (Aldrich et al., 1979). We have shown that, in voltageclamped bag cell neurons, the delayed outward currents also inactivate with repetition in a frequency-dependent manner (Strumwasser et al., 1981) and our preliminary data indicate that these outward currents are diminished further by $8 \mathrm{BT}-\mathrm{cAMP}$. Such further inactivation of outward potassium currents, therefore, probably underlies the effects of 8BT-cAMP on the width of bag cell action potentials. A long lasting inactivation of potassium currents also could explain the ability of repetitive action

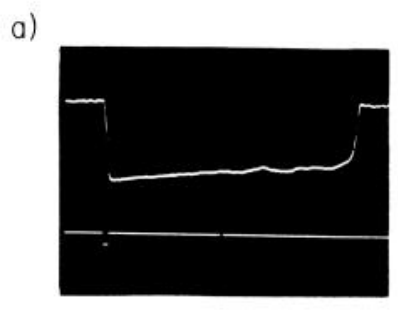

b)

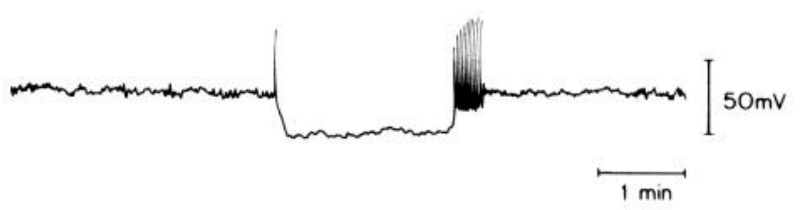

Figure 7. A regenerative hyperpolarizing response observed in cultured bag cells following 8BT-cAMP (0.5 mM). $a$, The left panel shows the regenerative hyperpolarizing response (upper trace) triggered by a brief hyperpolarizing current pulse (lower trace). The right panel shows the response of the membrane potential to multiple current pulses. The third pulse shown is of greater magnitude than the remainder and triggers the regenerative hyperpolarizing response. During the regenerative response, the membrane potential excursions evoked by the subthreshold current pulses are reduced markedly relative to the control, indicating increased membrane conductance. $b$, Two examples of spontaneously occurring regenerative hyperpolarizing responses in $8 \mathrm{BT}$-cAMP-treated cultured bag cells. 
A.

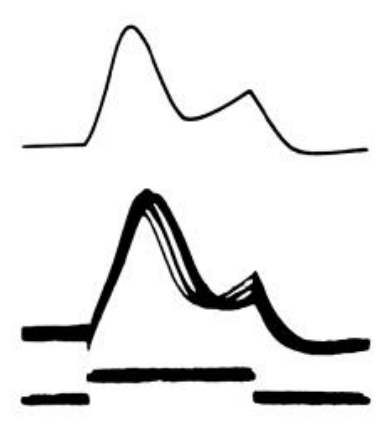

B.

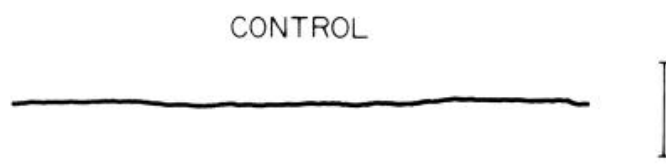

8BT CAMP INJECTED

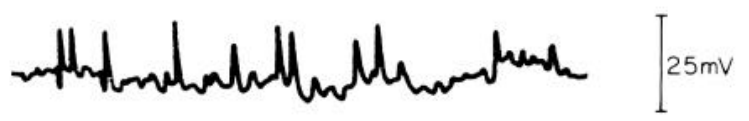

I min

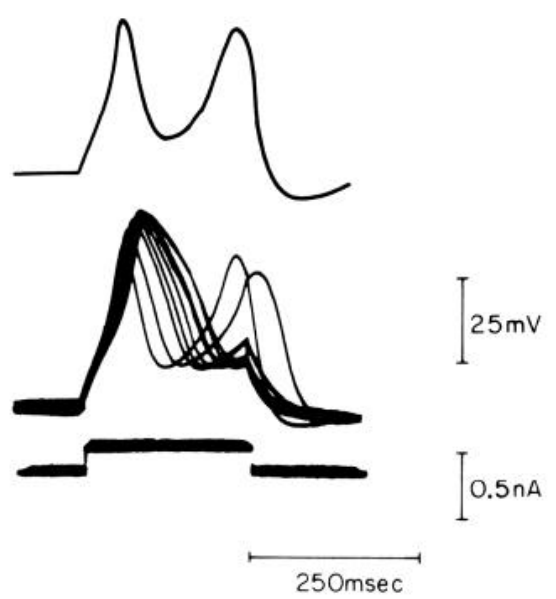

C.

APPLIED

CURRENT ( $n A$ )
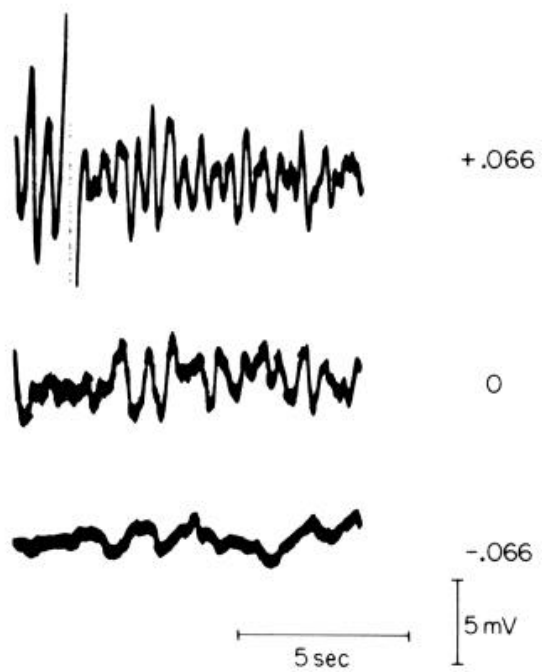

Figure 8. A, The effect of intracellular injection of 8BT-cAMP on bag cell action potentials and frequency-dependent spike broadening. The uppermost traces show the voltage response to the first depolarizing current pulse in a train of 10 pulses applied at a frequency of $1 / \mathrm{sec}$ before and after pressure injection of $8 \mathrm{BT}$-cAMP (17 psi, $30 \mathrm{sec}$ ). The middle set of traces shows the 10 superimposed action potentials evoked by the consecutive depolarizing current pulses (lower traces). B, The emergence of subthreshold oscillations in membrane potential and discharge following injection of 8BT-cAMP (21 psi, 2 sec). $C$, Oscilloscope tracings of subthreshold oscillations that persisted in a cultured bag cell neuron penetrated with a large tipped $8 \mathrm{BT}$-cAMPcontaining microelectrode. Depolarizing current increased the frequency and amplitude of the oscillations (upper trace), whereas hyperpolarizing current diminished the oscillations (lower trace).

potentials to trigger prolonged afterdischarges in those experiments where a hyperpolarizing bias current prevented spontaneous discharge.

It is also possible that 8BT-cAMP has direct effects on inward calcium currents. The action potentials of bag cell neurons in cell culture, as in the intact bag cell cluster, may have either mixed sodium-calcium components or pure calcium components (Acosta-Urquidi and Dudek, 1979; Kaczmarek et al., 1980; Strumwasser et al., 1981). Cells, such as those used for the extracellular application of 8BT-cAMP, with higher resting potentials and well defined thresholds, may have appreciable so- dium components to their action potentials (Strumwasser et al., 1981). On the other hand, we have shown that cells with lower resting potentials, such as those used for intracellular injection, may have action potentials that are totally insensitive to the sodium channel blocker, tetrodotoxin, but which may be abolished by the calcium antagonist, $\mathrm{Co}^{2+}$ (Kaczmarek et al., 1980). In these latter cells, injection of 8BT-cAMP (see "Results") or of the catalytic subunit of cAMP-dependent protein kinase (Kaczmarek et al., 1980) also may increase the amplitude and slope of the rising phase of the calcium action potentials. Although this effect could, conceivably, result from 
a modification of potassium channels by the cAMP analogue or by direct phosphorylation of substrate proteins by the kinase, it also could represent a direct enhancement of inward calcium currents. If an enhancement of calcium currents occurred in the cells used in the extracellular application studies, it is likely that the sodium component of their action potentials would mask such effects in voltage recordings. Whether there is, in fact, an effect on calcium currents as well as on potassium currents can be resolved only by further voltage clamp analyses of these cultured neurons.

Our evidence suggests that the regenerative hyperpolarizing response that was observed, in a minority of our experiments, following $8 \mathrm{BT}$-cAMP is most likely due to a transient increase in membrane potassium conductance. We have not observed spontaneous or evoked hyperpolarizing responses of this sort in intracellular recordings from intact bag cell clusters either in their resting or afterdischarging states. An interesting possibility, is that the response represents a transient return of the cells from a cAMP-activated, low potassium conductance state to the higher potassium conductance "resting" state. A similar hyperpolarizing response has been described in the R15 neuron of Aplysia where it can last routinely for more than 10 min (Strumwasser, 1973).

Effects similar to those that we have detailed for the peptidergic bag cell neurons have been described for a number of other types of molluscan neurons when treated with cAMP analogues or phosphodiesterase inhibitors (Gillette et al., 1978; Klein and Kandel, 1978; Treistman and Levitan, 1976). As one example, Gillette et al. (1978) showed that endogenous bursting and spike broadening are enhanced by these agents in the ventral white cells, which drive feeding behavior in Pleurobranchaea. These authors suggested that cAMP alters either the availability or the ability of internal $\mathrm{Ca}^{2+}$ ions to activate a $\mathrm{Ca}^{2+}$ dependent $\mathrm{K}^{+}$conductance. Broadening of the spikes by cAMP in sensory neurons appears to underlie the enhancement of transmitter release during sensitization of the gill withdrawal reflex in Aplysia (Klein and Kandel, 1978). It remains to be determined whether the biochemical and ionic sequelae to an increase in cAMP are identical in these different types of neurons.

\section{References}

Acosta-Urquidi, J., and F. E. Dudek (1979) Soma spike of neuroendocrine bag cells of Aplysia californica. Soc. Neurosci. Abstr. 5: 239.

Aldrich, R. W., P. A. Getting, and S. H. Thompson (1979) Mechanism of frequency-dependent broadening of molluscan neurone soma spikes. J. Physiol. (Lond.) 291: 507-530.

Blankenship, J. E., and J. T. Haskins (1979) Electronic coupling among neuroendocrine cells in Aplysia. J. Neurophysiol. 42: $347-355$.

Dudek, F. E., and J. E. Blankenship (1977a) Neuroendocrine cells of Aplysia brasiliana. I. Bag cell action potentials and afterdischarge. J. Neurophysiol. 40: 1301-1311.

Dudek, F. E., and J. E. Blankenship (1977b) Neuroendocrine cells of Aplysia brasiliana. II. Bag cell prepotentials and potentiation. J. Neurophysiol. 40: 1312-132 t.

Gillette, M. U., R. Gillette, and W. J. Davis (1978) Cyclic AMP may modulate prolonged endogenous bursting and spike broadening in the ventral white cell of Pleurobranchaea californica. Soc. Neurosci. Abstr. 4: 381.

Heller, E., L. K. Kaczmarek, M. Hunkapiller, and F. Strumwasser (1979) Afterdischarge in bag cell neurons is initiated by peptides from the atrial gland of Aplysia. Soc. Neurosci. Abstr. 5: 248.

Heller, E., L. K. Kaczmarek, M. W. Hunkapiller, L. E. Hood, and F. Strumwasser (1980) Purification and primary structure of two neuroactive peptides that cause bag cell afterdischarge and egg laying in Aplysia. Proc. Natl. Acad. Sci. U. S. A. 77: 2328-2332.

Kaczmarek, L. K., K. Jennings, and F. Strumwasser (1978) Neurotransmitter modulation, phosphodiesterase inhibitor effects, and CAMP correlates of afterdischarge in peptidergic neurites. Proc. Natl. Acad. Sci. U. S. A. 75: 5200-5204.

Kaczmarek, L. K., M. Finbow, J. P. Revel, and F. Strumwasser (1979) The morphology and coupling of Aplysia bag cells within the abdominal ganglion and in cell culture. J. Neurobiol. 10: 535-550.

Kaczmarek, L. K., K. R. Jennings, F. Strumwasser, A. C. Nairn, U. Walter, F. D. Wilson, and P. Greengard (1980) Microinjection of catalytic subunit of cyclic AMP-dependent protein kinase enhances calcium action potentials of bag cell neurons in cell culture. Proc. Natl. Acad. Sci. U. S. A. 77: 7487-7491.

Klein, M., and E. R. Kandel (1978) Presynaptic modulation of voltage-dependent $\mathrm{Ca}^{2+}$ current: Mechanism for behavioural sensitization in Aplysia californica. Proc. Natl. Acad. Sci. U. S. A. 75: 3512-3516.

Kupfermann, I. (1970) Stimulation of egg laying by extracts of neuroendocrine cells (bag cells) of abdominal ganglion of Aplysia. J. Neurophysiol. 33: 877-881.

Kupfermann, I., and E. R. Kandel (1970) Electrophysiological properties and functional interconnections of two symmetrical neurosecretory clusters (bag cells) in abdominal ganglion of Aplysia. J. Neurophysiol. 33: 865-876.

Leibovitz, A. (1963) The growth and maintenance of tissue-cell cultures in free gas exchange with the atmosphere. Am. J. Hyg. 78: 173-180.

McCaman, R. E., D. G. McKenna, and J. K. Ono (1977) A pressure system for intracellular and extracellular ejections of picoliter volumes. Brain Res. 136: 141-147.

Meyer, R. B., and J. P. Miller (1974) Analogs of cyclic AMP and cyclic GMP: General methods of synthesis and the relationship of structure to enzymic activity. Life Sci. 14: 1019-1040.

Strumwasser, F. (1973) Neural and humoral factors in the temporal organization of behavior. Physiologist 16: 9-42.

Strumwasser, F., L. K. Kaczmarek, and D. Viele (1978) The peptidergic bag cell neurons of Aplysia: Morphological and electrophysiological studies of dissociated cells in tissue culture. Soc. Neurosci. Abstr. 4: 207.

Strumwasser, F., L. K. Kaczmarek, A. Y. Chiu, E. Heller, K. R. Jennings, and D. P. Viele (1980) Peptides controlling behavior in Aplysia. In Peptides: Integrators of Cell and Tissue Functions, F. E. Bloom, ed., pp. 197-218, Raven Press, New York.

Strumwasser, F., L. K. Kaczmarek, K. R. Jennings, and A. Y. Chiu (1981) Studies of a model peptidergic neuronal system, the bag cells of Aplysia. In Neurosecretion-Molecules, Cells, Systems, D. S. Farner and K. Lederis, eds., Plenum Press, NY, in press.

Stuart, D. K., A. Y. Chiu, and F. Strumwasser (1980) Neurosecretion of egg-laying hormone and other peptides from electrically active bag cell neurons of Aplysia. J. Neurophysiol. 43: $488-498$.

Treistman, S. N., and I. B. Levitan (1976) Alteration of electrical activity in molluscan neurons by cyclic nucleotides and peptide factors. Nature 261: 62-64. 